\title{
Mass drug administration programme against lymphatic filariasis-an evaluation of coverage and compliance in a northern Karnataka district, India
}

\author{
Praveen Kulkarni ${ }^{\mathrm{a}}$, Jose Jom Thomas ${ }^{\mathrm{a}, *}$, Jenee Dowerah ${ }^{\mathrm{a}}$, M.R. Narayana Murthy ${ }^{\mathrm{a}}$, \\ K. Ravikumar ${ }^{\mathrm{b}}$

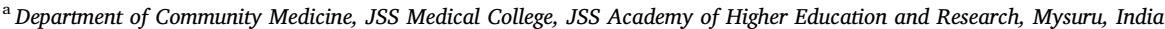 \\ ${ }^{\mathrm{b}}$ Department of Health and Family Welfare, Govt. of India, India
}

\section{A R T I C L E I N F O}

\section{Keywords:}

Lymphatic filariasis

Compliance

Coverage

MDA

Bagalkot district

\begin{abstract}
A B S T R A C T
Background and objectives: Lymphatic Filariasis, also known as elephantiasis, is a neglected tropical disease causing chronic damage to the lymphatic system. The disease, due to its peculiar morphological manifestations and disfigurations, is associated with severe social stigma and results in severe economic and mental stress for the affected. India's National Health Policy (2002) was aiming at the elimination of Lymphatic Filariasis in the country by 2015 which was extended to 2017. Preventive chemotherapy using yearly one dose of DEC and albendazole through mass drug administration is the key strategy adopted for this. This study aims at assessing the coverage, compliance, and causes for noncompliance towards MDA in Bagalkot district and assessing the rates of directly observed treatment, the source of information on MDA and incidence of side effects related to MDA Programme.

Methods: A cross-sectional study was done in four randomly selected clusters in endemic Talukas of Bagalkot district of Karnataka. A total of 240 households with 1110 beneficiaries were evaluated during the study. The information was collected by directly interviewing one responsible adult member from each house using a structured proforma. The data obtained was then entered to Microsoft Excel 2013 spreadsheet and analyzed using SPSS version 22.

Results: Among the 1110 persons evaluated the coverage was found to be $93.9 \%$ and effective coverage rate (compliance) was $83.2 \%$. The coverage compliance gap was $10.7 \%$. The most common reason quoted by the beneficiaries for not consuming the tablet was lack of faith in the tablets. The incidence of adverse drug reactions was $14.68 \%$.

Conclusion: The effective coverage rate of MDA programme in Bagalkot was well below the national target of $85 \%$, recommended by the government of India. The major reason for the high coverage-compliance gap was the failure of effective IEC activities. Intensive IEC activities are needed for educating the beneficiaries against the misconceptions of filariasis and MDA programme.
\end{abstract}

\section{Introduction}

Lymphatic filariasis, also known as elephantiasis, is a neglected tropical disease causing chronic damage to the lymphatic system and transmitted through mosquitoes. ${ }^{1}$ Infection by nematodes of the family Filariodea belonging to three species Wuchereria bancrofti, Brugia malayi, and Brugia timori leads to this condition. The disease is mainly transmitted by Culex mosquito which is seen widely across urban and semi-urban areas and occasionally by Anopheles and Aedes mosquitoes. ${ }^{2}$ The majority of cases of lymphatic filariasis are asymptomatic.
However, it can cause chronic damage to the lymphatic, immune and secretory system of the body without any manifestation. The symptomatic variety of disease can have acute or chronic manifestations like inflammation of the skin, lymph nodes and lymphatic vessels and/or lymphedema or elephantiasis of limbs, breasts, and genitals. The disease due to its peculiar morphological manifestations and disfigurations is associated with severe social stigma and results in severe economic and mental stress for the affected. ${ }^{1,3}$

Filariasis is a major economic and social problem in tropical and subtropical Africa, Asia, Western Pacific and parts of America. World

\footnotetext{
${ }^{*}$ Corresponding author. Department of Community Medicine, JSS Medical College, JSS Academy of Higher Education and Research, Mysuru, Karnataka, India.

E-mail address: josejom031@gmail.com (J.J. Thomas).
} 
Health Organization states that currently, 856 million people in 52 countries are living in areas at risk where preventive measures should be taken to control the disease. It is estimated that at least 25 million men are affected by hydrocele and 15 million people by lymphedema. $90 \%$ of lymphatic filariasis infection is caused by W. bancrofti alone.1 South East Asia due to its geo-climatic conditions possesses the majority of lymphatic filariasis cases. According to the Ninth Meeting of the Regional Programme Review Group (RPRG), Yangon, Myanmar, 30th April-1st May 2012, 50\% of people affected globally reside in South East Asia and $63 \%$ of people at risk of developing lymphatic filariasis are in 9 out of 11 SEAR countries (Bangladesh, India, Indonesia, Maldives, Myanmar, Nepal, Sri Lanka, Thailand and Timor-Leste). ${ }^{4}$

In India, the disease is endemic in 256 districts distributed in 16 States and 5 UTs. The population at risk is about 630 million. Except for the North-Western States/UTs namely Jammu \& Kashmir, Himachal Pradesh, Punjab, Haryana, Chandigarh, Rajasthan, Delhi and Uttaranchal, and the North-Eastern States namely Sikkim, Arunachal Pradesh, Nagaland, Meghalaya, Mizoram, Manipur and Tripura, all other states in the country are endemic for Filariasis. ${ }^{5}$

In Karnataka, 13.28 Million people live in the endemic area of which 10.14 million are from rural areas. Six districts namely Bagalkot, Bidar, Bijapur, Gulbarga, Raichur, are considered as endemic for Filariasis. 25 filaria clinics, 8 filaria control units and 1 survey unit functioning in 6 districts of karntaka. ${ }^{5}$

Being a signatory to the World Health Assembly Resolution in 1997 for Global Elimination of Lymphatic Filariasis, India's National Health Policy (2002) was aiming at the elimination of Lymphatic Filariasis in the country by 2015. The Global goal for elimination is by 2020. In 2004, the Indian government launched The National Elimination of Lymphatic Filariasis Programme with preventive chemotherapy through annual Mass Drug Administration (MDA) as the key strategy. MDA was observed with a single drug - DEC initially, but since 200607, co-administration of DEC + Albendazole were introduced for Drug Administration. ${ }^{6}$

The government of India provided the logistic support to all the endemic states and UTs in terms of training material, drugs and a cash grant for programme implementation on an annual basis. Each year the Centre provides a media campaign and advocacy and IEC prototype materials are additionally provided to States and UTs for its dissemination. The current first pillar of strategy for elimination of the disease is Annual Mass Drug Administration (MDA) of single dose of DEC (Diethylcarbamazine citrate) and Albendazole for minimum of 5 years or more to the eligible population at risk (except pregnant women, children below 2 years of age and seriously ill persons) to interrupt transmission of the lymphatic filariasis. Home-based management of chronic cases and upscaling of selected CHCs, district hospitals and medical colleges for hydrocele operations are also part of the strategy. ${ }^{6}$

Bagalkot, being an endemic district in Karnataka, had observed 15th round of Mass Drug Administration in the year 2018. With the instructions from the Regional Health Office, Government of India, Bangalore, Department of Community Medicine, JSS Medical College, Mysore conducted the evaluation of MDA programme in the district on 29th of October 2018. The objectives of the study were to assess the coverage and compliance towards mass drug administration in the district and to assess the rates of directly observed treatment, the source of information on MDA and incidence of side effects related to MDA Programme.

\section{Methods}

This cross-sectional study was conducted in the month of October 2018. The guidelines for selection of clusters and size of population to be covered in each cluster was issued by the ministry of health and family welfare, government of India. According to these guidelines total four clusters were selected from the district of which one was in the urban and three were in the rural settings. ${ }^{7}$ Multistage sampling technique was followed. Badami and Hunagund were two Talukas which were endemic for lymphatic filariasis in the district. One Rural primary health centre from Badami taluka and two rural primary health centres from Hunagund taluka were selected randomly by lottery method. From each of these PHCs one Sub Centre was selected randomly by lottery method and a village under it was selected by the same method for the study. For the selection of urban cluster, all the wards in Badami town were line listed and one ward was randomly selected by lottery method. The primary health centres selected were Guledgudda (urban), Katageri, Hiresinganagutti, and Kandagal. Investigators visited selected villages and a centre point of the village was identified with the help of local residents. From the centre, four directions were identified and numbered. One direction was chosen randomly for the survey and the number of houses in the direction was noted. Investigators then did a house to house survey in the street and one adult member within the age group 18-60 years was selected from each house for the survey. The data regarding demographic profile, awareness about elephantiasis, coverage, compliance, adverse drug reactions, etc. was obtained by direct interview technique using a structured proforma after explaining the purpose of the survey and obtaining verbal consent. A flashcard containing a picture of elephantiasis case, DEC and albendazole tablets was used to explain about the survey to participants. Totally 240 houses were covered with 60 houses in each of the clusters.

The data collected was entered in Microsoft Excel spreadsheet and analyzed with SPSS version 22 licensed to JSS Academy of Higher Education and Research. Percentages were used for descriptive data and relevant statistical tests were done to find association. The results were expressed with descriptive tables and relevant pictorial representations.

\section{Results}

Within 240 houses visited 1110 persons were identified as beneficiaries after excluding children less than 2 years, pregnant women and elderly persons with chronic diseases. Among the beneficiaries, 525 (47.3\%) were males and $585(52.7 \%)$ were females. $20.18 \%$ of the samples studied were children less than 15 years.

1042 persons among the total 1110 samples studied had received DEC and Albendazole tablets as part of mass drug evaluation programme giving a coverage of $93.9 \%$. In the sample studied, 923 had consumed the tablets distributed to them and the effective coverage rate (ECR) or compliance estimated was $83.2 \%$ in Bagalkot district.

The four clusters studied were comprised of one urban and three rural clusters with a total of 267 samples from the urban area (24.05\%) and $843(75.95 \%)$ from rural areas. The coverage in the rural area (95.1\%) was significantly higher than coverage in the urban area $(89.9 \%)$ with a chi-square test $p$ value of 0.002 . Effective coverage rate or compliance of the population towards the MDA program was higher in rural areas (87.9\%) compared to the urban area (68.2\%). This was significant with a $\mathrm{p}$ value less than 0.001 in chi-square test. The total coverage compliance gap (CCG) in Bagalkot district was estimated to be $10.7 \%$ with a $21.7 \%$ CCG in the urban area and $7.2 \%$ in rural areas. (Table 1).

The only source of information about the MDA programme and the preventive chemotherapy among the beneficiaries was the drug distributors including ASHA workers and Anganwadi workers. None of the beneficiaries reported to knew about the program through any IEC activities. $82.1 \%$ of participants responded that the drug distributor explained about the disease, reasons for MDA and possible side effects of the disease while remaining $17.39 \%$ were unaware of the above mentioned. Only 74.1 of beneficiaries consumed the tablets in the presence of drug distributor (Directly Observed Treatment).

The main reasons for not consuming the tablets among the respondents the lack of faith in the tablets, belief that tablets are not required if not affected by the disease and being out of station during 
Table 1

The coverage rate, compliance (Effective Coverage rate) and Coverage- Compliance gap of mass drug administration programme in rural and urban areas of Bagalkot district.

\begin{tabular}{|c|c|c|c|c|c|}
\hline Locality & Coverage Rate & $\mathrm{P}$ value & ECR (Compliance rate) & $P$ value & Coverage Compliance gap \\
\hline Urban & $240(89.9 \%)$ & 0.002 & $182(68.2 \%)$ & $<0.001$ & $21.7 \%$ \\
\hline Rural & $802(95.1 \%)$ & & $741(87.9 \%)$ & & $7.2 \%$ \\
\hline Total & $1042(93.9 \%)$ & & $923(83.2 \%)$ & & $10.7 \%$ \\
\hline
\end{tabular}

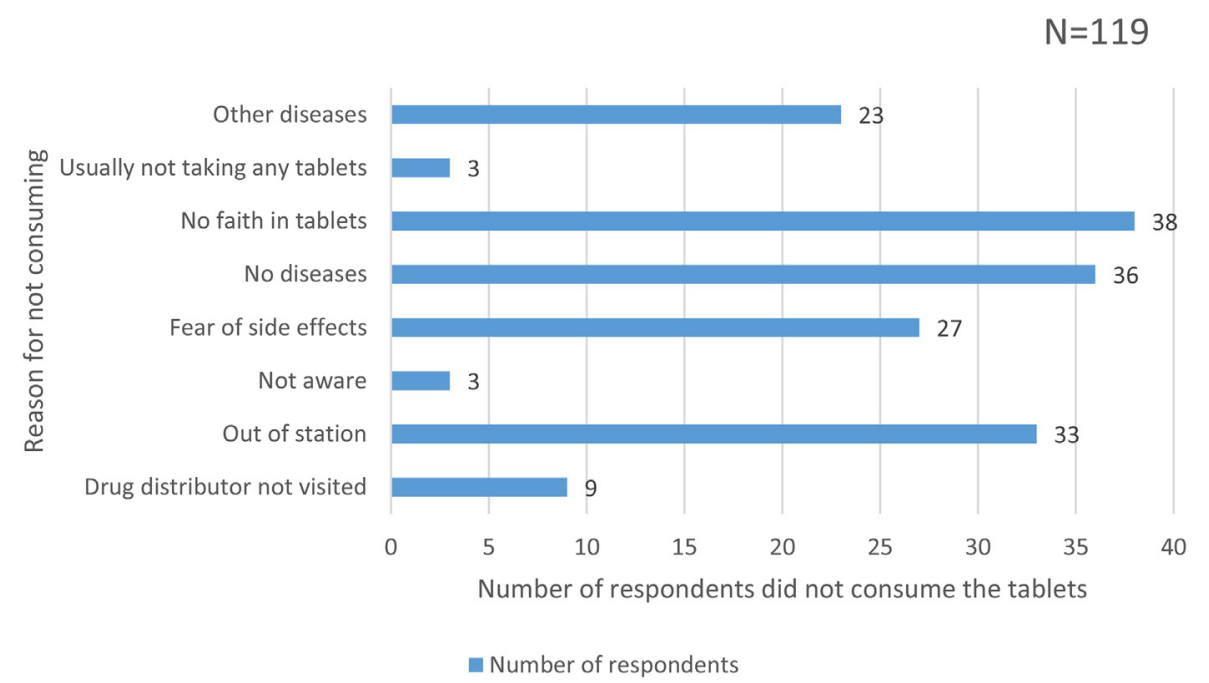

Fig. 1. Bar chart showing reasons of not consuming the preventive chemotherapy tablets among the respondents.

the visit by drug distributor (Fig. 1).

The incidence of adverse drug reactions in our study was $14.68 \%$. 163 beneficiaries out 1110 samples studied developed minor side reactions. Most common side effect reported was fever (96.53\%). Other side reactions include Sedation (1.73\%), Diarrhoea (0.58\%), Headache $(0.58 \%)$, and fatigue $(0.58 \%)$. All the events were self-limiting lasting for less than $24 \mathrm{~h}$ and none of the victims had to take any medications to limit the side reactions.

\section{Discussion}

The present study is a cross-sectional study done in October 2018 with objectives to assess the coverage and compliance towards mass drug administration in the district and to assess the rates of directly observed treatment, the source of information on MDA and incidence of side effects related to MDA programme. The coverage of mass drug administration observed in Bagalkot district was $93.9 \%$ with compliance a rate (effective coverage rate- ECR) of $83.2 \%$. The coverage compliance gap observed was $10.7 \%$. However, the coverage obtained in the district was lower than the national expected standards (minimum 85\% ECR) to interrupt the transmission and eliminate the disease in a particular area. ${ }^{6}$

Compared to the previous scenario, the coverage obtained by our study in 2018 in Bagalkot district is higher. A study done by Gudegowda KS et al. in Bagalkot in 2016 observed a coverage rate of $88.5 \%$ which is lesser than the coverage obtained in the present study which is $93.9 \% .^{8}$ Another study conducted in 2010 in the same district shows a coverage rate of $78.6 \% .^{9}$ However considering the coverage compliance gap an effective coverage rate of only $83.2 \%$ was observed in the present study which is lesser than observed by Gudegowda KS et al., in 2016.7 Similar study conducted in Bijapur district by Kulkarni $P$ et al., in 2017 shows a coverage rate of $80.3 \%$ and effective coverage rate of $72.5 \%$ and Waseem A et al., in 2014 shows a coverage and compliance rate of $81.63 \%$ and $79.21 \%$ respectively. ${ }^{10,11}$

Similar to a previous study conducted by Kulkarni et al. in Bijapur district in 2017, the coverage and compliance were significantly higher in rural areas compared to urban areas. Hence it proves the good impact of village level health workers like Anganwadi workers and ASHAs in mobilizing community towards MDA, which is specifically lacking in urban areas. ${ }^{10}$

The mass drug administration program recommends that the administration of drugs should be under direct observation of the drug distributor. $74.1 \%$ of beneficiaries reported that they followed this in our study. This was higher than the observations in the previous years. $^{8,10}$

The only source of information about MDA program, need for chemotherapy and possible side effects to the beneficiaries in surveyed clusters of Bagalkot district was the drug distributors (ASHA workers and Anganwadi workers). None of the participants reported any IEC materials as the source of information in contrast to other studies. The coverage-compliance gap compared to a similar study conducted in the same area in 2016 was 0.96 . The higher coverage-compliance gap we observed $(10.7 \%)$ in contrast to the above mentioned study can be attributed to weak IEC activities in the district. ${ }^{8}$

The three most common reasons for not consuming the tablets according to the survey were the lack of faith in the tablets, belief that tablets are not required if not affected by the disease (lack of awareness) and being out of station during the visit by drug distributor. In an older study conducted by Patel PK in 2010, major reasons for nonconsumption of the drugs were not receiving the tablet, not present at home when the drug distributor visited and unawareness about the tablets in Bagalkot district. Another study by Waseem A et al. in the neighboring district of Bijapur revealed the fear of side reactions as the main reasons for not adhering to the preventive chemotherapy. A similar study conducted by Kulkarni et al. also shows lack of awareness and fear of side reactions as the two major causes of not consuming the tablets. $^{9-11}$

In contrast to a similar study done in the same district in 2016 where none of the beneficiaries developed side reactions, we observed an incidence of $14.68 \%$ of side reactions after the MDA programme. ${ }^{8}$ 
The incidence of side reactions was higher according to our study in contrast to similar studies conducted before like Kulkarni et al. (2.4\%), Hoolageri MS et al. (4.1\%). ${ }^{10,12}$ A notably different pattern of side reactions was observed in the study with the major pattern of side effects being hyperpyrexia $(96.53 \%)$ in contrast to previous studies were nausea and vomiting being the major side reaction of mass drug administration. ${ }^{10,12}$

\section{Conclusion}

Our study concluded that the coverage rate of Mass Drug Administration programme to prevent lymphatic filariasis in Bagalkot district of Karnataka was $93.9 \%$ with an effective coverage rate or compliance of $83.2 \%$. The coverage-compliance gap in the district was estimated to be $10.7 \%$. The coverage and compliance were significantly higher in rural areas compared to urban areas with a low coveragecompliance gap in rural areas. Drug distributors were the only source of information in all the clusters evaluated. The main reasons for not consuming the tablets among the respondents the lack of faith in the tablets, belief that tablets are not required if not affected by the disease and being out of station during the visit by drug distributor. The incidence of adverse drug reactions after consuming the tablets was $14.68 \%$ and all the reactions were self-limiting.

\section{Conflicts of interest}

None.

\section{Ethical issues}

Nil.

\section{References}

1. Park K. Park's Textbook of Preventive and Social Medicine. 24th ed. Jabalpur: M/s Banarsidas Bhanot; 2017:287 (Chapter 5), Epidemiology of Communicable Diseases.

2. World Health Organization. Lymphatic filariasis. . [Internet]. [cited 2019 Feb 01]. Available from:. https://www.who.int/lymphatic filariasis/en/.

3. Suryakantha AH. Community Medicine with Recent Advances. fourth ed. New Delhi: The Health Sciences publisher; 2017:455-462 Section 5, (Chapter 20) (Epidemiology of communicable diseases).

4. World Health Organization. Elimination of Lymphatic Filariasis in the South-East Asia Region: Report of the Ninth Meeting of the Regional Programme Review Group (RPRG) Yangon, Myanmar. WHO Regional Office for South-East Asia; 2012.

5. NVBDCP. National vector borne disease control programme. Available at: http:// www.nvbdcp.gov.in/filariasis.html, Accessed date: 1 February 2018.

6. Elimination of Lymphatic Filariasis. Training Manual on Mass Drug Administration and Morbidity Management. New Delhi: Ministry of Health and Family Welfare, NVBDCP, Government of India; 2004.

7. WHO. Coverage Evaluation Surveys for Preventive Chemotherapy: Field Guide for Implementation.

8. Gudegowda KS, Duraisamy S, Narayanappa RR, Sobagiah RT. Evaluation of mass drug administration for lymphatic filariasis in Bagalkot district, Karnataka, cross sectional study. Natl J Res Community Med. 2017;6(1):71-76.

9. Patel PK. Mass drug administration coverage evaluation survey for lymphatic Filariasis in Bagalkot and Gulbarga districts. Indian J Community Med: official publication of Indian Association of Preventive \& Social Medicine. 2012 Apr;37(2):101.

10. Kulkarni P, Krishnaveni YS, Murthy MN, Kumar KR. Coverage and compliance towards mass drug administration programme against lymphatic filariasis in

Vijayapura (Bijapur) district, Karnataka, India. International Journal Of Community Medicine And Public Health. 2018 Sep 24;5(10):4311-4315.

11. Waseem A, Dorle AS, Mannapur BS, Vetri S. Coverage and compliance of mass drug administration for elimination of lymphatic filariasis in Bijapur District, Karnataka. Annals of Community Health. 2015 Dec 31;2(4):6-10.

12. Hoolageri MS, Kamath R, Ravikumar K, Jagadish G, Kamath S. Evaluation of mass drug administration programme for elimination of lymphatic filariasis in Bidar district, Karnataka. International Journal Of Community Medicine And Public Health. 2018 Feb 24;5(3):1020-1023. 